\title{
CLASSIFICATION OF SURFACES
}

\author{
JUSTIN HUANG
}

ABSTRACT. We will classify compact, connected surfaces into three classes: the sphere, the connected sum of tori, and the connected sum of projective planes.

\section{Contents}

1. Introduction

2. Topology

3. Complexes and Surfaces

4. Classification of Surfaces

5. The Euler Characteristic

6. Application of the Euler Characterstic

\section{INTRODUCTION}

This paper explores the subject of compact 2-manifolds, or surfaces. We begin with a brief overview of useful topological concepts in Section 2 and move on to an exploration of surfaces in Section 3. A few results on compact, connected surfaces brings us to the classification of surfaces into three elementary types. In Section 4, we prove Thm 4.1, which states that the only compact, connected surfaces are the sphere, connected sums of tori, and connected sums of projective planes. The Euler characteristic, explored in Section 5, is used to prove Thm 5.7, which extends this result by stating that these elementary types are distinct. We conclude with an application of the Euler characteristic as an approach to solving the map-coloring problem in Section 6. We closely follow the text, Topology of Surfaces, although we provide alternative proofs to some of the theorems.

\section{TOPOLOGY}

Before we begin our discussion of surfaces, we first need to recall a few definitions from topology.

Definition 2.1. A continuous and invertible function $f: X \rightarrow Y$ such that its inverse, $f^{-1}$, is also continuous is a homeomorphism. In this case, the two spaces $X$ and $Y$ are topologically equivalent.

Date: August 15, 2008. 
This definition of a homeomorphism means that $f$ is a continuous bijection such that $f$ maps open sets to open sets. We also have the following notions of compactness and connectedness.

Definition 2.2. An open cover of a subset $A$ of the topological space $X$ is a collection $\vartheta$ of open subsets of $X$ such that $A \subseteq \bigcup_{O \in \vartheta} O$. A subcover of $\vartheta$ is a subcollection $\vartheta^{\prime} \subseteq \vartheta$ that is an open cover of $A$. A finite subcover is a finite subcollection that is an open cover of $A$.

Definition 2.3. A topological space $X$ is compact if every open cover of $X$ has a finite subcover.

Definition 2.4. A topological space $X$ is disconnected if there exist two non-empty disjoint open sets $U_{1}, U_{2}$ such that $X=U_{1} \cup U_{2}$. A topological space $X$ is connected if it is not disconnected.

Examples 2.5. The following sets are illustrations of these two concepts.

(1) The unit interval is compact and connected.

(2) The real numbers are not compact, but connected.

(3) The set of two elements $\{0,1\}$ using the discrete topology is compact but not connected.

(4) The integers, using the discrete topology, are not compact and not connected.

Later in the paper, we will use various manipulations to classify and reduce compact, connected surfaces. The following notions of the quotient topology and quotient space are signficant, then, because they allow us to cut and glue spaces together to form new spaces.

Definition 2.6. Given a function $f: X \rightarrow Y$, where $X$ is a topological space and $Y$ is a set, the quotient topology on $Y$ is such that $U \subseteq Y$ is open if and only if $f^{-1}(U)$ is open in $X$.

Definition 2.7. Given $X$ a topological space and $x \sim y$ an equivalence relation, the quotient space is the set of equivalence classes $X / \sim:=\{[x]: x \in X\}$.

Examples 2.8. Quotient Spaces.

(1) $\mathbb{S}^{1}=[0,1] /\{0,1\}$.

(2) The Mobius band $=\{(x, y): 0 \leq x \leq 1,0 \leq y \leq 1\} /(0, y) \sim(1,1-y)$.

(3) $\mathbb{S}^{2}=D^{2} / \mathbb{S}^{1}$.

(4) $\mathbb{P}^{2}=\mathbb{R}^{3}-0 / \sim$, where $x \sim \lambda x$ for $\lambda \in \mathbb{R}$. $\mathbb{P}^{2}$, the projective plane, cannot be embedded in 3 dimensions.
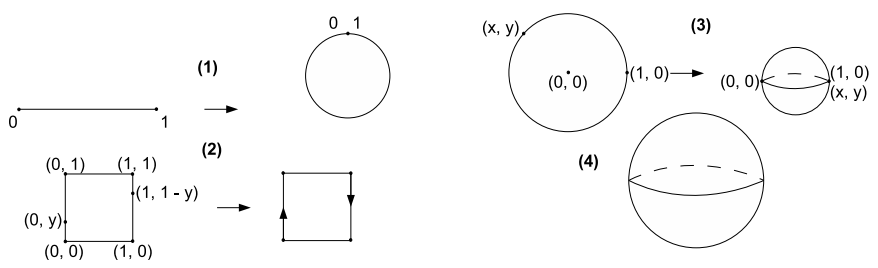

Figure 1. The quotient spaces of Example 2.8 


\section{Complexes and Surfaces}

We begin this section with a series of defintions working our way up to surfaces. We then show that surfaces can be represented by planar diagrams, providing a relatively simple way of manipulating surfaces.

Definition 3.1. A cell is a space whose interior is homeomorphic to the unit $n$-dimensional ball of the Euclidean space $\mathbb{R}^{n}$. The boundary of a cell must be composed of a finite number of lower-dimensional cells, the faces of the cell, where 0, 1, 2, 3-dimensional cells are as defined below:

(1) A 0-dimensional cell is a point or vertex (normally labelled with a capital letter)

(2) A 1-dimensional cell is a line segment AB starting and ending at two 0dimensional cells $(\mathrm{A}, \mathrm{B})$. Note that vertices $\mathrm{A}$ and $\mathrm{B}$ are faces of $\mathrm{AB}$

(3) A 2-dimensional cell is a polygon of line segments. For example, the quadrilateral defined by the line segments AB, BC, CD, and DA. Note that line segments AB, BC, CD, DA are faces of the quadrilateral ABCD, as well as A, B, C, D.

(4) A 3-dimensional cell is a solid polyhedron of polygons. Note again that vertices, line segments, and polygons are all the faces of the polyhedron.

Definition 3.2. A complex $K$ is a finite set of cells with the following properties:

(1) The faces of the cells of $K$ are also cells of $K$.

(2) If $\sigma$ and $\tau$ are cells in $K$, then $\operatorname{int}(\sigma) \cap \operatorname{int}(\tau)=\emptyset$.

A complex where all cells have dimension at most $n$ is an $n$-complex. For $K$ a complex, we write $|K|$ to denote the geometry resulting from gluing cells together.

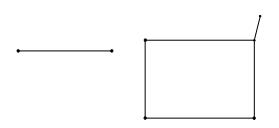

Figure 2. Two complexes. The first is a 1-complex, the second a 2-complex.

Definition 3.3. A planar diagram is a polygon with $2 n$ edges where pairs of edges are identified with either the same or opposite orientation. Given the quotient topology, the labelled edges are identified or glued together.

Planar diagrams are 2-complexes that code the gluing instructions needed for the construction of 2-manifolds. Below is an example of a planar diagram.

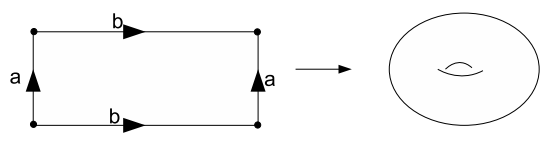

Figure 3. The planar diagram of and surface represented by $a b a^{-1} b^{-1}$. 
Definition 3.4. An n-dimensional manifold is a topological space satisfying two properties:

(1) every point has a neighborhood that is topologically equivalent to an $n$ dimensional open disc.

(2) any two distinct points can be contained by disjoint neighborhoods.

A 2-manifold is a surface. We will be dealing with compact and connected surfaces in our classification of surfaces.

Example 3.5. The following are compact, connected surfaces along with common planar diagram representations.

(1) $\mathbb{S}^{2}$ the sphere in $\mathbb{R}^{3}$ represented by the circle in $\mathbb{R}^{2}$ with the upper hemisphere edge identified with the lower hemisphere edge.

(2) $\mathbb{T}^{2}$ the torus in $\mathbb{R}^{3}$ represented by the two pairs of opposite edges identified together.

(3) $\mathbb{P}^{2}$ the projected plane in $\mathbb{R}^{4}$ represented by circle in $\mathbb{R}^{2}$ with the upper hemisphere edge identified opposingly with the lower hemisphere edge. $\mathbb{P}^{2}$ can also be defined in terms of the quotient topology, as above.
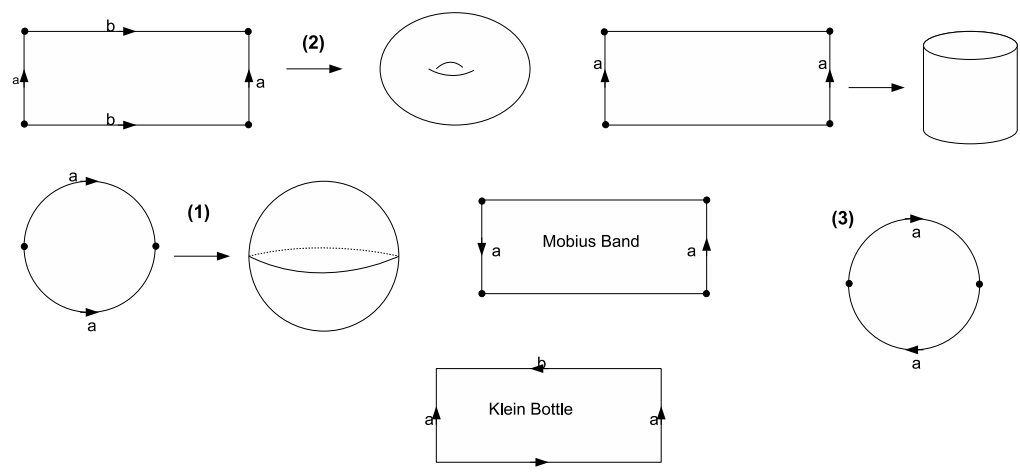

Figure 4. The planar diagrams of some important compact, connected surfaces, the sphere, the torus, the projective plane. The cylinder, Mobius band, and Klein bottle are also included.

The neighborhoods of points on the planar diagram can be used to check whether a space is a surface, as illustrated in the following theorem.
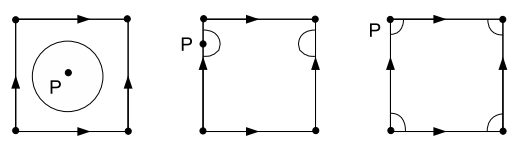

FIGURE 5. The neighborhoods of an interior point, an edge point, and a vertex point of a planar diagram.

Theorem 3.6. The topological space represented by a planar diagram is a compact, connected surface. 
Proof. Figure 5 shows that every point has a neighborhood topologically equivalent to a 2-dimensional open disc. The figure also demonstrates that the space is Hausdorff. Using the quotient topology, we have a continuous function mapping the planar diagram to the topological space. Since the planar diagram is compact and connected, we have that the space is compact and connected.

Definition 3.7. A 2-dimensional topological space $X$ is triangulable if a 2-complex structure $K$ can be found with $X=|K|$ where $K$ only has triangular cells and identification between triangles occurs only along a single edge or a single vertex. Such a $K$ is called a triangluation of $X$. A triangulated surface is a surface $X$ with a triangulation.

By cutting and gluing, we can combine two surfaces to form a new surface using the quotient topology.

Definition 3.8. A connected sum is the new surface formed by combining two or more compact, connected surfaces in the following manner. Remove small discs from $S_{1}, S_{2}$, the two original surfaces, and glue the boundary circles together. When dealing with triangulated surfaces, we can take the discs to be triangles. We write $S_{1} \# S_{2}$ for the connected sum of two surfaces and $n S$ for $S \# S \# \cdots \# S n$ times.

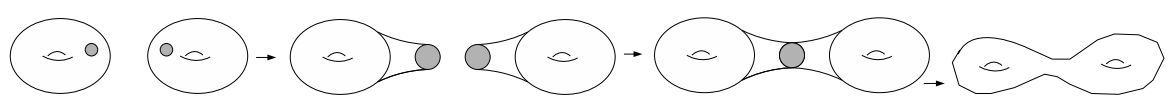

Figure 6. The connected sum of two tori.

With the understanding of surfaces given above, we can now begin to show how any compact, connected surface can be homeomorphically reduced to three elementary types of surfaces, the primary goal of this paper. The following lemmas will justify the steps of our proof of Thm 4.1.

Lemma 3.9. If a set $D$ has a discrete topology, then $D$ is compact if and only if $D$ is finite.

Proof. This proof follows from clever choices of open covers.

Given $D$ compact and the discrete topology, we choose an open cover $\left\{U_{\alpha}\right\}_{\alpha \in I}$ such that only one point of $D$ is in $U_{\alpha}$. Since $D$ is compact, we have a finite subcover of this cover, say $U_{\alpha_{1}}, \ldots, U_{\alpha_{n}}$. Since these sets cover $D$ and there are finitely many of them, $D$ is finite.

Let $D$ be finite. If $\left\{U_{\alpha}\right\}_{\alpha \in I}$ is an open cover, for each $d_{i} \in D$, let $d_{i} \in U_{\alpha_{i}}$. Then, $U_{\alpha_{1}}, \ldots, U_{\alpha_{n}}$ is an open subcover. Thus, $D$ is compact.

Lemma 3.10. Let $P$ be a set consisting of one interior point from each triangle in a finite triangulation. Then $P$ is closed and discrete.

Proof. It is clear that $P$ is closed. $P$ is discrete by considering the interior of each triangle.

Lemma 3.11. Every compact, connected surface can be represented as a planar diagram.

Proof. We will prove the following two results. 
(1) A surface is compact if and only if any triangulation uses a finite number of triangles.

(2) A surface is connected if and only if a triangulation can be arranged in order $T_{1}, T_{2}, \ldots T_{n}$ with each triangle having at least one edge identified to an edge of a triangle listed earlier.

With these two results, we have an ordered list of triangles with which to work. Placing the triangles in order as listed, we see we have both inner and outer edges. These inner edges are already identified together and so we need only consider outer edges. Thus we have a polygon with labelled edges that, when correctly identified, form a surface. In other words, we have a planar diagram, as desired.

(1) A surface is compact if and only if any triangulation uses a finite number of triangles.

Take any arbitrary triangulation. In order to show that it uses a finite number of triangles, it suffices to show either that $P$, the set of one interior point for each triangle, is finite. From Lemma 3.10, $P$ is compact, since closed subsets of compact sets are compact, and discrete. Then by Lemma $3.9, P$ is finite.

Take an open cover of a triangulation. Given finitely many triangles in all triangulations, it is easy to show that each triangle has a finite subcover. It then follows that we have a finite subcover for the entire space.

(2) A surface is connected if and only if a triangulation can be arranged in order $T_{1}, T_{2}, \ldots T_{n}$ with each triangle having at least one edge identified to an edge of a triangle listed earlier.

Assuming $S$ is connected, we will relabel the triangles so that at least one edge of a triangle is identified to an edge of a triangle listed earlier. Start with the triangle $T_{1}$. Since $S$ is connected, there exists a $T_{2}$ glued to $T_{1}$ along an edge. Let our inductive hypothesis be that there are $n-1$ triangles arranged as desired. We then need only to glue a triangle $T_{n}$. Assume we cannot. Then $S$ can be expressed as the disjoint union of $\bigcup_{i=1}^{k-1} T_{i}$ and $T_{k}$. This contradicts $S$ being connected, and so we have that $T_{n}$ can be connected to the list of triangles $T_{n-1}$. In other words, we have ordered triangles $T_{1}, \ldots T_{n}$ as desired.

Given an ordered triangulation, suppose that $S$ is not connected. Then $S$ has at least two connected parts. Let the first part be $T_{1}, T_{2}, \ldots, T_{k}$ and the second part $T_{k+1}, T_{k+2}, \ldots, T_{m}$. Because these are disjoint parts of $S$, $T_{k+1}$ cannot be connected to any of $T_{1}, T_{2}, \ldots, T_{k}$, thus contradicting the ordered triangulation of the hypothesis. We then conclude that our original assumption was incorrect, and that $S$ is connected.

Lemma 3.12. The following manipulations of planar diagrams do not change the homeomorphism type of the surfaces the diagrams represent. The lower-case letters $a$ or $b$ are single edges. $w$ is a word, that is, a sequence of letters grouped together.

(1) Renaming edges. $w_{1} w w_{2} w w_{3}=w_{1} c w_{2} c w_{3}$ or $w_{1} w w_{2} w^{-1} w_{3}=w_{1} c w_{2} c^{-1} w_{3}$.

(2) Collapsing edges. $w_{1} a a^{-1} w_{2}=w_{1} w_{2}$

(3) General starting point. $w_{1} w_{2}=w_{2} w_{1}$ 
(4) Cutting and gluing. Cutting is of the form $w_{1} w_{2} w_{3} w_{4}=\left(w_{1} w_{2} a\right.$ and $\left.a w_{3} w_{4}\right)$, where $a$ is the cut edge. Gluing along $a$ is of the form $\left(w_{1} w_{2} a\right.$ and $\left.a w_{3} w_{4}\right)=w_{1} w_{2} w_{3} w_{4}$ or $\left(w_{1} w_{2} a\right.$ and $\left.w_{4} a^{-1} w_{3}\right)=w_{1} w_{2} w_{4} w_{3}$.

Proof. We need to show that the changes undergone in the planar diagram represent homeomorphic changes undergone in the surface. Note: for the remainder of the paper, the planar diagrams will be composed of three types of lines. Continuous lines for single edges, dashed lines for cut edges, and double-dotted-and-dashed lines for words.

(1) This is easily a homeomorphism since it is merely a relabelling of the representative planar diagram.

(2) Figure 7 shows that this step is a homeomorphism.

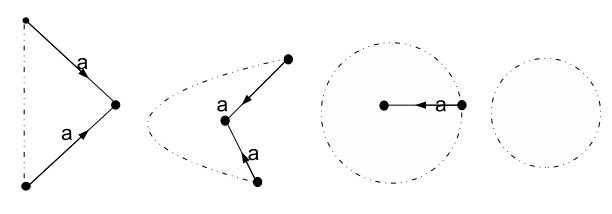

FiguRE 7. Illustration of the homeomorphism, collapsing.

(3) This is easily a homeomorphism since it is merely a relabelling of the representative planar diagram.

(4) It suffices to check continuity for points on the cut edge. We have that the map is a local homeomorphism by seeing we have half disc neighborhoods at the two cut edges. When re-glued, these are full discs. Figure 8 illustrates this proof.

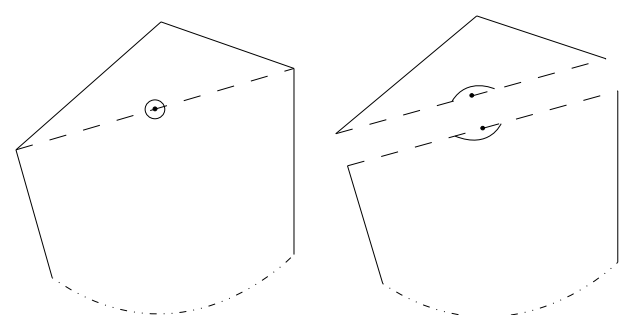

Figure 8. The neighborhood around a point along a cut edge remains a full disc.

\section{Classification of Surfaces}

In proving the Classification of Surfaces, we will assume the non-trivial result that all compact, connected surfaces allow a triangulation. With this result, we will be able to use the lemmas on triangulations proved in the previous section.

Theorem 4.1. Every compact connected surface is homeomorphic to a sphere, a connected sum of tori, or a connected sum of projective planes. 
Proof. The manipulations below are those from Lemma 3.12 and we have shown above that these represent homeomorphisms. All we need to show now is a constructive proof illustrating that the surfaces can be reduced to $\mathbb{S}^{2}$, connected sums of $\mathbb{T}^{2}$, or connected sums of $\mathbb{P}^{2}$. As a constructive proof, steps are provided for a methodological approach to this reduction. An example of this step-wise process is given after the proof.

\section{Step 1: A Planar Model}

Lemma 3.11 states that $\mathrm{S}$ has a planar diagram. Without loss of generality, using Lemma 3.12 (1), we can assume that there are no repeated words.

\section{Step 2: Adjacent Opposing Pairs}

Definition 4.2. A twisted pair of edges is a pair of the same, non-adjacent edges that point in the same direction, for example, ..a...a... An opposing pair of edges is a pair of the same, non-adjacent edges that point in 'opposing' directions, for example, $\ldots a \ldots a^{-1} \ldots$.

By Lemma 3.12 (2), without loss of generality, we have eliminated all adjacent opposing pairs. If all we had to begin with were adjacent opposing pairs, then we have $\mathbb{S}^{2}$.

\section{Step 3: Reduce to One Vertex}

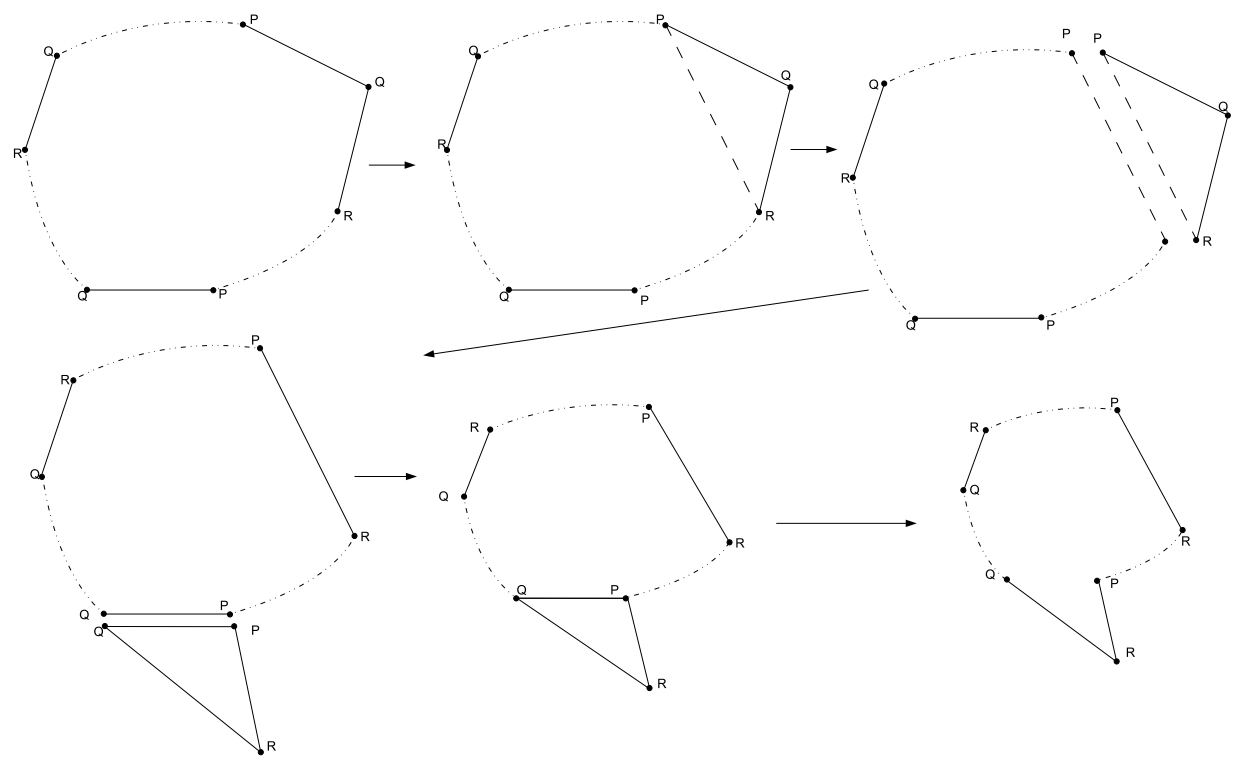

Figure 9 . We begin with $3 \mathrm{Q}$ vertices and $2 \mathrm{R}$ vertices. We end with $2 \mathrm{Q}$ vertice and $3 \mathrm{R}$ vertices. By induction, we result in only the $\mathrm{R}$ vertex.

We can now relabel the vertices. We do this by picking a vertex and calling it $\mathrm{P}$. Note that $\mathrm{P}$ begins an edge and ends an edge. Label every vertex that begins or ends these edges $\mathrm{P}$ and continue in this manner until all possible $\mathrm{P}$ vertices are labelled. If there are no more vertices, we have reduced the polygon to one vertex. If not, label vertices $\mathrm{Q}$ in a similar fashion and so on. 
We wish now to reduce the number of vertices to one without changing the homeomorphism type of the diagram. We employ Lemma 3.12 (4), the cutting and gluing step, to reduce $n$ vertices of an undesirable letter to $n-1$ vertices of that letter and $k$ vertices of a desirable letter to $k+1$ vertices of that letter. The steps are illustrated in Figure 9. By induction, we continue this process until only one vertex remains.

\section{Step 4: Collecting Twisted Pairs}

We again employ cutting and gluing multiple times to bring all twisted pairs together, as shown in Figure 10.
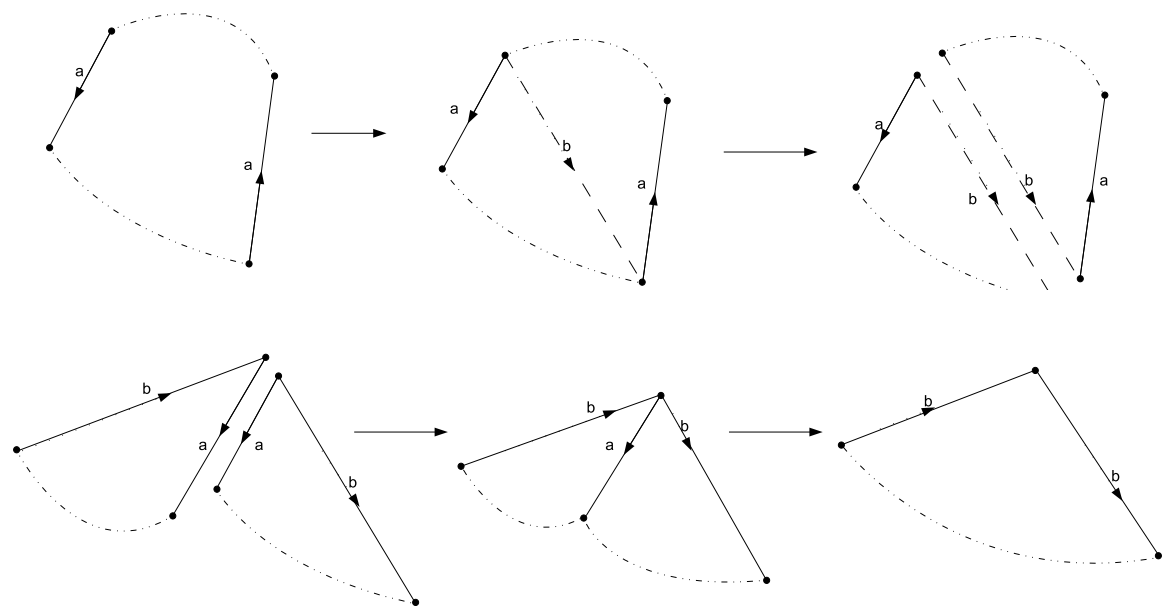

FiguRE 10. Bringing non-adjacent twisted pairs together.

We continue by induction, and if the only edges that remain are adjacent twisted pairs, then the surface is $n \mathbb{P}^{2}$.

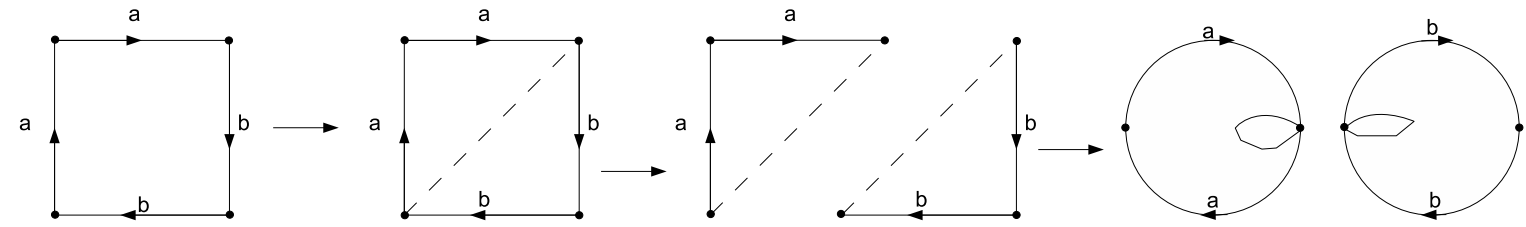

FiguRE 11. A word consisting of adjacent twisted pairs is the connected sum of multiple projective planes.

\section{Step 5: Collecting Pairs of Opposing Pairs}

Given Steps 1 through 4, we know that these pairs of opposing pairs exist.

The alternative, that is, a single opposing pair, means that the remaining edges will be twisted pairs. We then have a word of the form $w_{1} a w_{2} a^{-1}$ where $w_{1}$ and $w_{2}$ are words consisting of adjacent twisted pairs. We will see that if Step 3 were carried out, then this is impossible, that is, we will find more than one vertex. Label the vertex between $w_{1}$ and $a P$. $P$ begins $a$ and is found throughout $w_{1}$ since 

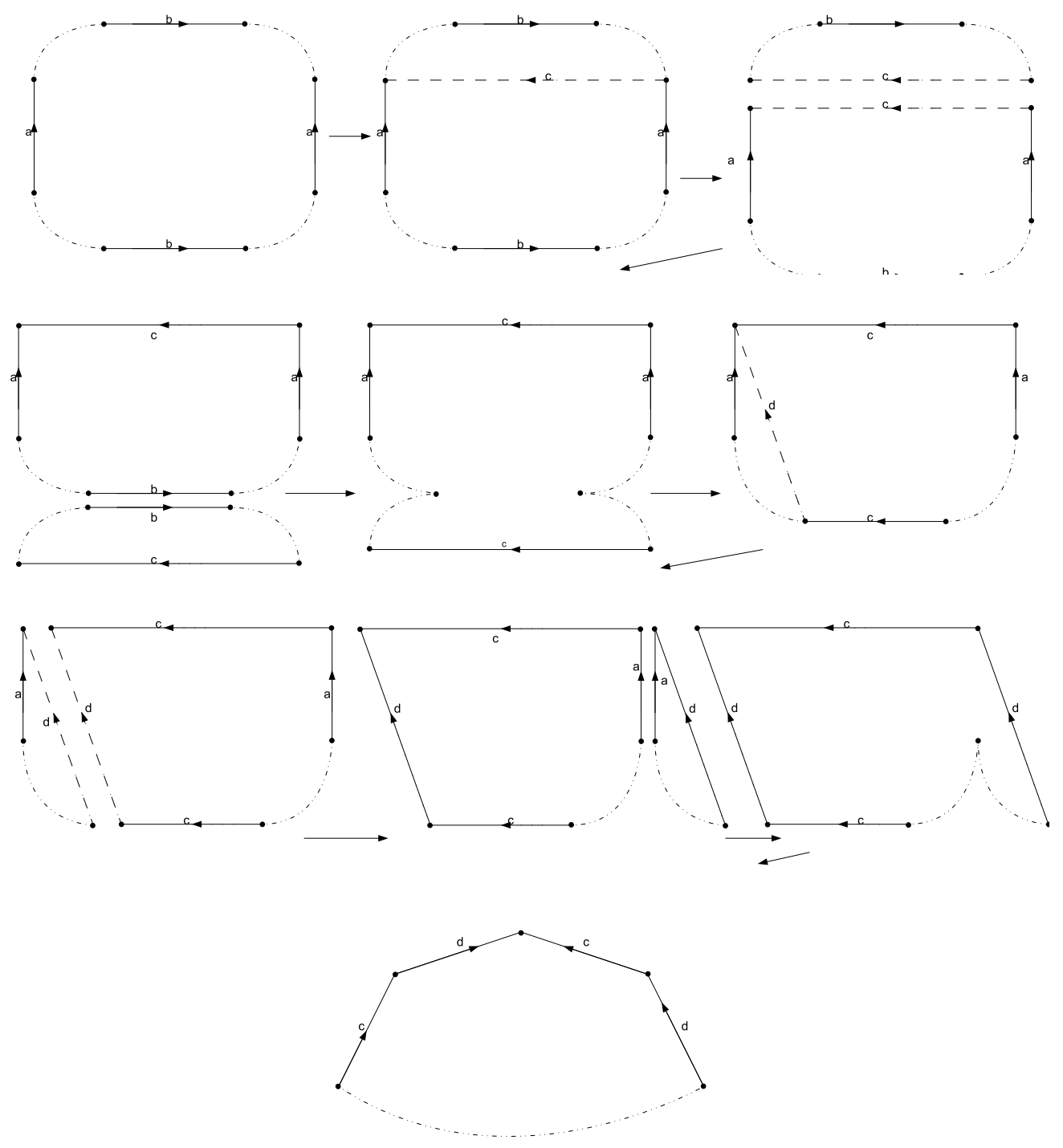

Figure 12. Manipulating a Pair of Opposing Pairs to form $c d c^{-1} d^{-1}$.

$w_{1}$ consists of adjacent twisted pairs, where there are vertices both beginning and ending the same edge. Since no edge in $w_{1}$ is identified to an edge in $w_{2}$, we have to start labelling with another vertex, $Q$, and so we have clearly not carried out Step 3.

We perform a series of cutting and gluing maneuvers to bring pairs of opposing pairs into the form $a b a^{-1} b^{-1}$. By induction, we have combinations of adjacent opposing pairs and adjacent twisted pairs.

Step 6: $\mathbb{T}^{2}$ and $\mathbb{P}^{2}$ At this point we either have $\mathbb{S}^{2}$, connected sums of $\mathbb{T}^{2}$, or a planar diagram with twisted pairs and pairs of opposing pairs. The following lemma shows that these can be reduced to twisted pairs. 
Lemma 4.3. The connected sums $\mathbb{T}^{2} \# \mathbb{P}^{2}$ and $\mathbb{P}^{2} \# \mathbb{P}^{2} \# \mathbb{P}^{2}$ are homeomorphic.
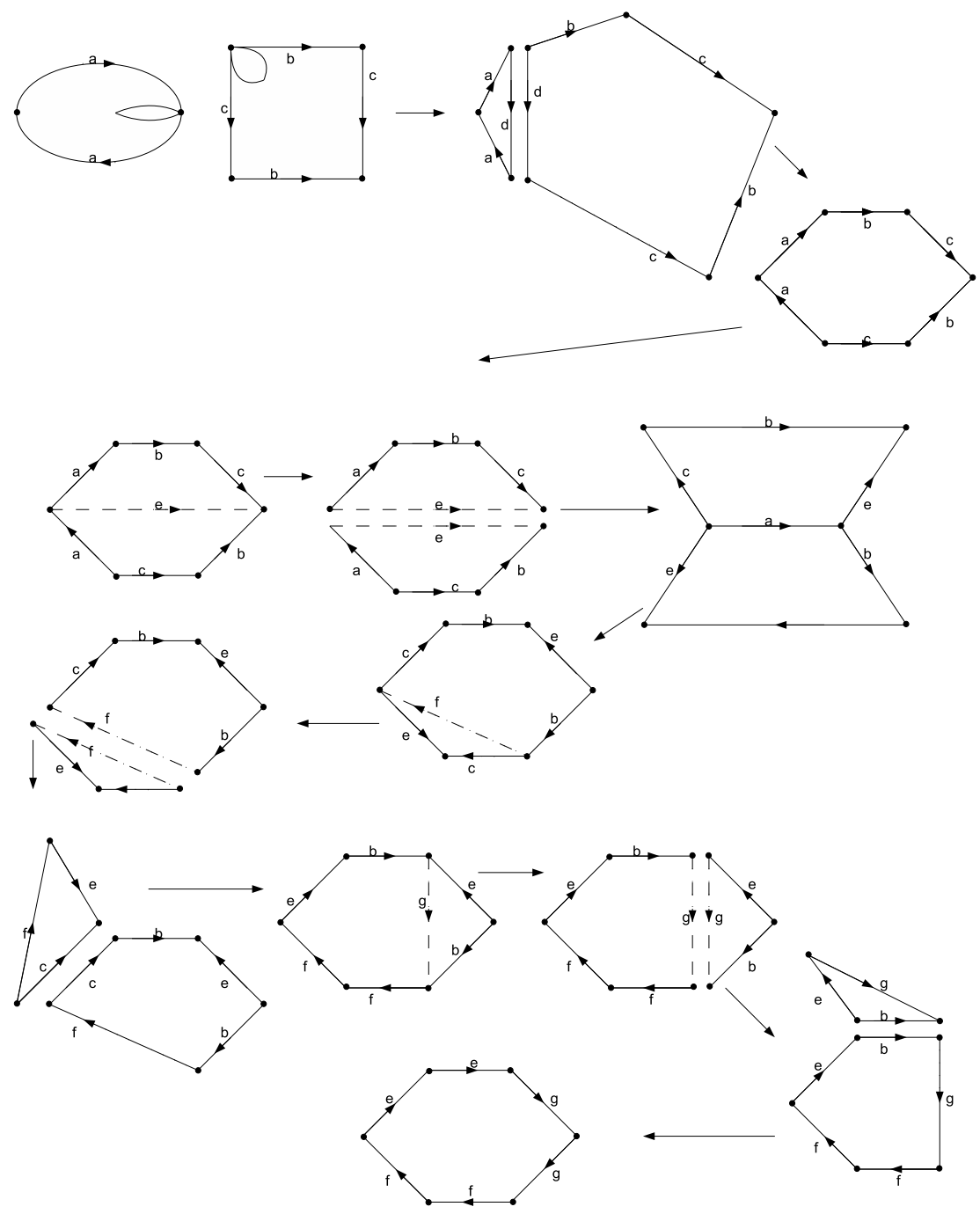

Figure 13. The reduction of $\mathbb{T}^{2} \# \mathbb{P}^{2}$ to $\mathbb{P}^{2} \# \mathbb{P}^{2} \# \mathbb{P}^{2}$.

Proof. This proof begins with the planar model of the torus and the projected plane. Through a series of clever cuts and glues as shown in Figure 13, we arrive at the connected sum of three projected planes.

After these steps have been executed in order, we have found either the sphere, the connected sum of tori, or the connected sum of projective plane as the classification of our unknown surface. 


\section{The Euler Characteristic}

We have accomplished the first task of finding elementary forms under which all surfaces can be classified: the sphere, the connected sum of tori, and the connected sum of projective planes. However, the complementary task of ensuring that any two of these elementary forms are not homeomorphic remains. We now turn to the Euler characteristic to further differentiate surfaces with very little information - namely, the underlying structure defined by vertices, edges, and faces of planar diagrams.

Definition 5.1. A quantity $\alpha$ is a topological invariant if $\alpha(X)=\alpha(Y)$ whenever $X$ and $Y$ are topologically equivalent.

A significant topological invariant is the Euler characteristic.

Definition 5.2. The Euler characteristic of a finite complex $K$ is

$$
\chi(K)=\sum_{j=0}^{\infty}(-1)^{j} \#(j-\text { cell })
$$

where $(j-$ cell $)$ refers to the number of n-cells in the complex $K$.

If we focus on 2-complexes, that is, surfaces, as we have throughout this paper, we can restrict the Euler characteristic to surfaces and examine the equation

$$
\chi(K)=v(K)-e(K)+f(K)
$$

where $v(K)$ is the number of vertices of $K, e(K)$ the number of edges of $K$, and $f(K)$ the number of faces of $K$.

Theorem 5.3. If $|K|$ and $|L|$ are compact, connected surfaces and $|K| \cong|L|$, then $\chi(K)=\chi(L)$.

Proof. This proof closely follows the proof to Thm 4.1 and shows that each of the types of manipulations taken by the proof results in no change in the Euler characteristic. In each case, a step will take a complex $K$ to a complex $L$ with cells $f, e, v$ of $K$ and cells $f^{\prime}, e^{\prime}, v^{\prime}$ of $L$. The types of steps are triangulation, adding an edge between two vertices, adding a vertex in the interior of a polygon and an edge from the new vertex to an original vertex, and adding a vertex in the interior of an edge. These types were noted throughout the proof of Thm 4.1.

(1) Type 1: Adding an edge between two vertices of a polygon. We have that $f^{\prime}=f+1, e^{\prime}=e+1$, and $v^{\prime}=v$, so that

$$
\chi(L)=f^{\prime}-e^{\prime}+v^{\prime}=(f+1)-(e+1)+v=f-e+v=\chi(K)
$$

This type of move occurs in Steps 3, 4, and 5 of Thm 4.1.

(2) Type 2: Adding a vertex in the interior and an edge from a new vertex to a boundary vertex of a polygon. We have that $f^{\prime}=f, e^{\prime}=e+1$, and $v^{\prime}=v+1$, so that

$$
\chi(L)=f^{\prime}-e^{\prime}+v^{\prime}=f-(e+1)+(v+1)=\chi(K)
$$

This type of move occurs in Steps 1 and 2 of Thm 4.1.

(3) Type 3: Adding a vertex in the interior of an edge. We have that $f^{\prime}=f$, $e^{\prime}=e+1$, and $v^{\prime}=v+1$, so that, as above,

$$
\chi(L)=f^{\prime}-e^{\prime}+v^{\prime}=f-(e+1)+(v+1)=\chi(K)
$$

This type of move occurs in Step 1 of Thm 4.1. 
All of the steps of Thm 4.1 thus have no effect on the Euler characteristic. We now have that the Euler characteristic is invariant for compact, connected surfaces.

Definition 5.4. If $S$ is a compact, connected surface, and $S \cong|K|$ for a complex $K$, define $\chi(S)=\chi(K)$.

Thm 5.3 tells us that this is well-defined, and furthermore, that it is a topological invariant.

There is another powerful result of the Euler characteristic that relates the Euler characteristic of a connected sum of surfaces and the Euler characteristics of both surfaces.

Corollary 5.5. $\chi\left(S_{1} \# S_{2}\right)=\chi\left(S_{1}\right)+\chi\left(S_{2}\right)-2$.

Proof. Recall that connected sums are formed by removing discs from two surfaces and gluing the surfaces together at these removed discs. Without loss of generality, take triangulated complexes representing $S_{1}$ and $S_{2}, K_{1}$ and $K_{2}$. Take the removal of the discs to be removals of the faces of triangles on the complexes. We have $S_{1} \# S_{2}=\left(K_{1}-\right.$ triangle $) \cup\left(K_{2}-\right.$ triangle $)$ where the union is at the missing triangle. Call this complex $K$.

(1) the vertices of $K$ are $v(K)=v\left(K_{1}\right)+v\left(K_{2}\right)-3$.

(2) the edges of $K$ are $e(K)=e\left(K_{1}\right)+e\left(K_{2}\right)-3$.

(3) the faces of $K$ are $f(K)=f\left(K_{1}\right)+f\left(K_{2}\right)-2$.

Therefore, $\chi\left(S_{1} \# S_{2}\right)=\chi(K)=\chi\left(K_{1}\right)+\chi\left(K_{2}\right)-2$.

We now have had enough experience with the Euler characteristic to tackle the question that has provided the motivation for this section, namely, whether or not the elementary types of surfaces (the sphere, the connected sum of tori, and the connected sum of projective planes) are really different. We begin by calculating the Euler characteristic for these surfaces.

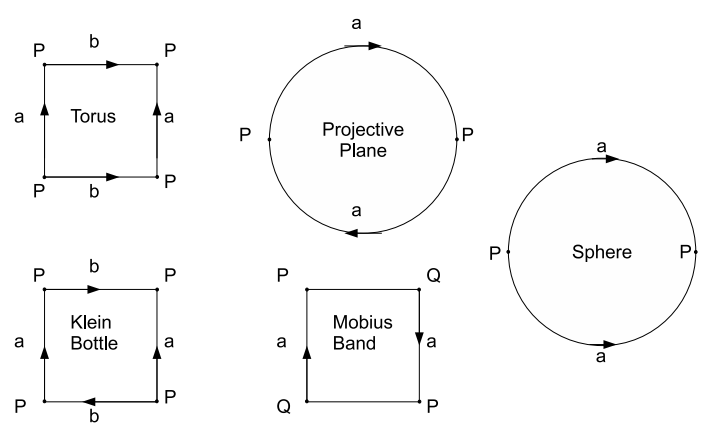

FiguRE 14. The planar diagrams of the sphere, torus, projective plane, Klein bottle, and Mobius band.

Example 5.6. What is the Euler characteristic for the torus, the projective plane, the Klein bottle, and the Mobius strip? We use the normal planar diagrams of these surfaces illustrated in Figure 14. 
(1) Looking at the planar diagram of the sphere, we see that it has two vertices $P$ and $Q$, one edge $a$, and one face. Thus, $\chi\left(\mathbb{S}^{2}\right)=2-1+1=2$.

(2) Looking at the planar diagram of the torus, we see that it has one vertex $P$, two edges $a$ and $b$, and one face. Thus, $\chi\left(\mathbb{T}^{2}\right)=1-2+1=0$.

(3) Looking at the planar diagram of the projective plane, we see that it has one vertex $P$, one edge $a$, and one face. Thus, $\chi\left(\mathbb{P}^{2}\right)=1-1+1=1$.

(4) Looking at the planar diagram of the Klein bottle, we see that it has one vertex $P$, two edges $a$ and $b$, and one face. Thus, $\chi($ Klein $)=1-2+1=0$.

(5) Looking at the planar diagram of the Mobius strip, we see that it has two vertices $P$ and $Q$, one edge $a$, and one face. Thus, $\chi($ Mobius $)=2-1+1=$ 2 .

Theorem 5.7. Every compact, connected surface is homeomorphic to exactly one of the following: $\mathbb{S}^{2}, n \mathbb{T}^{2}$, or $n \mathbb{P}^{2}$.

Proof. By Thm 4.1, it suffices to prove that $\mathbb{S}^{2}, n \mathbb{T}^{2}$, and $n \mathbb{P}^{2}$ are all topologically distinct. By Example 5.6 and Corollary 5.5, we have:

(1) $\chi\left(\mathbb{S}^{2}\right)=2$.

(2) $\chi\left(n \mathbb{T}^{2}\right)=2-2 n$.

(3) $\chi\left(n \mathbb{P}^{2}\right)=2-n$.

The only case of overlap, then, is when $\chi\left(n \mathbb{T}^{2}\right)=\chi\left(2 n \mathbb{P}^{2}\right)$. In other words, by Thm 5.3, we need to show that $n \mathbb{T}^{2}$ is not homeomorphic to $2 n \mathbb{P}^{2}$. To see this, we observe that $n \mathbb{T}^{2}$ has a continuous outward-pointing normal vector for $n \mathbb{T}^{2} \subseteq \mathbb{R}^{3}$, and so it cannot contain a Mobius band. However, $\mathbb{P}^{2}$ contains a Mobius band, and hence $\mathbb{P}^{2} \# S$ does as well for any surface $S$. In particular, $2 n \mathbb{P}^{2}$ contains a Mobius band, and therefore $n \mathbb{T}^{2}$ is not homeomorphic to $2 n \mathbb{P}^{2}$.

We now have the tools for identifying and distinguishing surfaces. With Thm 4.1, we have found that all surfaces can be reduced to one of three classes of surfaces. With Thm 5.3 and Thm 5.7, we have that these three classes of surfaces are the only distinct, compact, connected surfaces.

\section{Application of the Euler Characterstic}

As a conclusion to this paper, we give an application of the Euler characteristic.

A geographic map can be seen as a complex with vertices, edges, and faces. Take, for example, a map of the United States. Its edges are the borders between states, its vertices are the points where three or more states intersect, and its faces are the states themselves. The general problem is coloring the map.

Definition 6.1. A legal coloring of a map is one such that no two adjacent faces have the same color.

Lemma 6.2. Let $S$ be a surface, $S=|K|$ where the complex $K$ has e $(K)$ edges and $f(K)$ faces. If $\frac{2 e(K)}{f(K)}<N$, then $N$ colors are enough to color the map on $S$ determined by $K$.

Proof. Proof by Induction. Pick an integer $N>\frac{2 e(K)}{f(K)}$ for all complexes $K$. Induct on $\mathrm{f}(\mathrm{K})$, the number of faces of $K$. The initial cases, $f(K)<N$ are trivial because there are more colors than faces. Assume that we can color any map with $m$ faces with $N$ colors. We will prove that we can do the same for any map with $(m+1)$ 
faces. We have $N>\frac{2 e(K)}{m+1}$, so the average number of edges per polygon is less than $N$. Therefore, there exists a 2-cell (a polygon) in our complex with fewer than $N$ edges. Label this polygon $P$. Union $P$ with a neighbor 2-cell, $Q$ and ignore the edge between them. The complex now has $m$ faces, so it can be colored with $N$ colors. Color as our inductive hypothesis allows. Bring back the edge separating $P$ from $Q$ and remove the color from $P$. Since $P$ has less than $N$ neighbors, we can use a color not used on any of these neighbors.

Now we have a bound on the number of colors needed for a legal coloring of a map on a surface in terms of complexes on the surface. The following theorem shows the relation between $\frac{2 e}{f}$ and the Euler characteristic (a function of surfaces).

Lemma 6.3. Let $v(K)$ be the number of vertices of a complex $K$ and $e(K)$ the number of edges. Then, $v(K) \leq \frac{2 e(K)}{3}$

Proof. When we glue the polygons to form $K$, each vertex is the meeting point of at least three polygons. Therefore, there are at least three edges meeting at each vertex. Thus, $3 v(K) \leq$ number of vertices before assembly $=2 e(K)$, since before assembly, we have the same number of edges as vertices. Therefore, $v(K) \leq$ $\frac{2 e(K)}{3}$.

Theorem 6.4. For $S$ a surface and any complex $K$ on $S$ with e edges and $f$ faces,

$$
\frac{2 e(K)}{f(K)} \leq 6\left(1-\frac{\chi(S)}{f(K)}\right) \text {. }
$$

Proof. By Lemma 6.3, we now have $\chi(S)-f(K)=v(K)-e(K) \leq \frac{-e(K)}{3}$. Hence, $3(f(K)-\chi(S)) \geq e(K)$. Dividing by $f(K)$ on both sides gives the desired result.

Theorem 6.5. For a compact, connected surface $S$, let $N(S)$ be the minimal number of colors needed to color all maps on $S$. Then, $N\left(\mathbb{T}^{2}\right)=7$ and $N\left(\mathbb{P}^{2}\right)=6$.
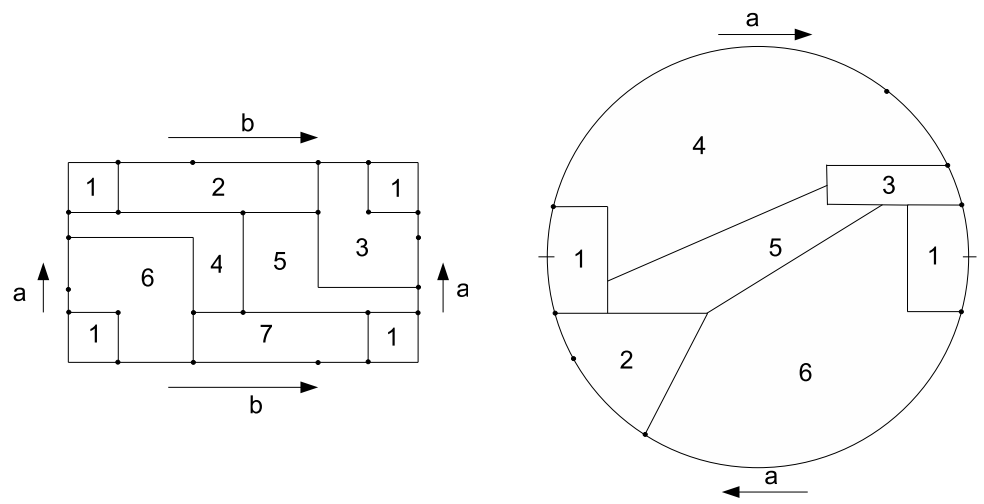

Figure 15. A map on the torus requiring seven colors and a map on the projective plane requiring six colors. The vertices are the vertices of the map or complex, not of the planar diagram. 
Proof. We show first that $N\left(\mathbb{T}^{2}\right) \leq 7$ and $N\left(\mathbb{P}^{2}\right) \leq 6$. For the torus, by Thm 6.4 , we have $\frac{2 e(K)}{f(K)} \leq 6$. Hence, by Lemma $6.2,7$ colors are sufficient to color any map on the torus. Similarly for the projective plane, $N \geq 6\left(1-\frac{1}{f(K)}\right)$. Thus, 6 colors are sufficient. We prove necessity by the maps on the torus and the projective plane shown in Figure 15. Note that every face is adjacent to every other face, and thus these maps illustrate necessity. We then have $N\left(\mathbb{T}^{2}\right)=7$ and $N\left(\mathbb{P}^{2}\right)=6$ as desired.

\section{REFERENCES}

[1] L. Christine Kinsey. Topology of Surfaces. Springer-Verlag New York, Inc. 1993. 